\title{
The impact of the initial wave of COVID-19 pandemic on children under endoscopic esophageal dilatation protocol: a single center study
}

\author{
Khaled Mohamed El-Asmar ${ }^{1} \cdot$ Mohammed Abdel-Latif $^{2}$ (D)
}

Received: 28 February 2021 / Revised: 12 April 2021 / Accepted: 17 April 2021 / Published online: 12 May 2021

(c) The Author(s), under exclusive licence to Springer Nature Singapore Pte Ltd 2021

\begin{abstract}
Purpose COVID-19 pandemic has adversely affected the medical services offered for non-COVID related pathologies all over the world. This led to most of the elective services being postponed. In this study, we investigated the impact of the initial wave of COVID-19 pandemic on patients with esophageal strictures that were listed on the endoscopic dilatation program. Methods Medical records were reviewed from March-September 2020 (study group). The study period was divided into three intervals guided by the number of confirmed cases with COVID-19 and the measures taken during the lockdowns in the corresponding periods. Case burden, dilatation sessions, dysphagia score, and further interventions were reviewed. Case burden and dilatation sessions were compared to equivalent periods in 2019 (control group).

Results The study group included 13 patients that had 19 dilatation sessions in contrast to 29 patients that had 98 sessions in the comparative group. In the study group, eight cases experienced deterioration in their dysphagia score, while further interventions were performed on four of them.

Conclusion To avoid morbidities during the pandemic, high-risk patients should be precisely identified and actively followed. Parents and caregivers should be reassured and encouraged to seek medical advice as quickly as possible whenever the need arises.
\end{abstract}

Keywords Esophageal stricture $\cdot$ Dysphagia $\cdot$ Covid-19 $\cdot$ Pediatric patients

\section{Introduction}

The current coronavirus disease (COVID-19) pandemic is challenging healthcare systems around the world. The World Health Organization (WHO) officially declared the disease an international emergency on March 11th, 2020 [1]. Routine medical practice has been greatly affected by the redistribution of resources, with the priority being given to COVID-19 infected patients.

Governments around the world have taken drastic measures to combat COVID-19. In addition, access to health services was severely affected due to the lockdown. These

Mohammed Abdel-Latif

m.latif@med.helwan.edu.eg

1 Pediatric Surgery Department, Ain Shams University, Cairo, Egypt

2 Pediatric Surgery Department, Helwan University, Cairo, Egypt lockdown measures raised concerns among the general population by negatively affecting their access to healthcare facilities.

Endoscopic esophageal dilatation for benign esophageal strictures is performed as part of routine work. Dilatation protocols for these patients aims at preserving their native esophagus. Loss of follow-up as well as interruptions in the dilatation program may have a negative impact on outcomes.

In the current study, the impact of the initial wave of the COVID-19 pandemic was evaluated in children, who previously experienced esophageal strictures, and were managed via a regular endoscopic dilatation protocol.

\section{Patients and methods}

In this study, the medical records of the Department of Pediatric Surgery at Ain Shams University were retrospectively reviewed for endoscopic esophageal dilatation during the initial wave of the COVID-19 pandemic, taking into account 
the associated period of lockdown. The analysis included patients having esophageal strictures from various etiologies and were being managed by the endoscopic dilatation protocol of Ain Shams University [2] from March 1st until the end of September 2020 (the study group).

\section{Dilatation protocol}

For caustic strictures, the dilation program was started six weeks after the incidence of alkali ingestion. For all benign esophageal strictures, including caustic strictures, an endoscopic dilatation session was attempted every two weeks for the first three months, then a session every month for the next three months, continued by a session every two months for the following six months. However, this program can be modified according to the patient's degree of dysphagia. In our center, we use the Savary-Gilliard bougie dilators ${ }^{\circledR}$ (Wilson-Cook Medical Inc., Winston-Salem, NC) under fluoroscopic guidance for endoscopic dilation. During the last decade, we have added topical Mitomycin $\mathrm{C}$ as an adjuvant therapy to decrease the incidence of re-fibrosis and recurrence [2].

Exclusion criteria were newly diagnosed cases of esophageal stricture as well as patients who underwent dilatation sessions due to the presence of neck anastomosis stricture after colon transposition.
Case burden and the number of dilatation sessions were examined during the study period. Patient demographic data, pathology, dysphagia score [3] and need for further interventions were reviewed.

The dysphagia score was based on the report published by Parthipun et al. [3], which classified dysphagia into five grades according to the ability to swallow: grade $0=$ normal diet, grade $1=$ swallow a semi-solid diet, grade $2=$ swallow a soft diet, grade $3=$ swallow liquids only and grade $4=$ complete dysphagia.

The study period was divided into 3-time intervals, guided by official numbers of infected cases announced by the Ministry of Health (Fig. 1) and measures taken during the first wave of the COVID-19 pandemic [4]. The first interval was beween March and April 2020 when there was a gradual increase in the number of infected cases, along with the initial measures of the lockdown. The second interval occurred during the months of May, June, and July when number of infected cases increased, despite the implementation of stricter lockdown measures. Whereas the third interval between August and September showed a gradual decrease in the number of infected cases, at a time when lockdown measures were being eased.

Data regarding case burden and number of dilatation sessions were compared to data recorded for equivalent periods in 2019 (the control group), after applying the same inclusion and exclusion criteria.

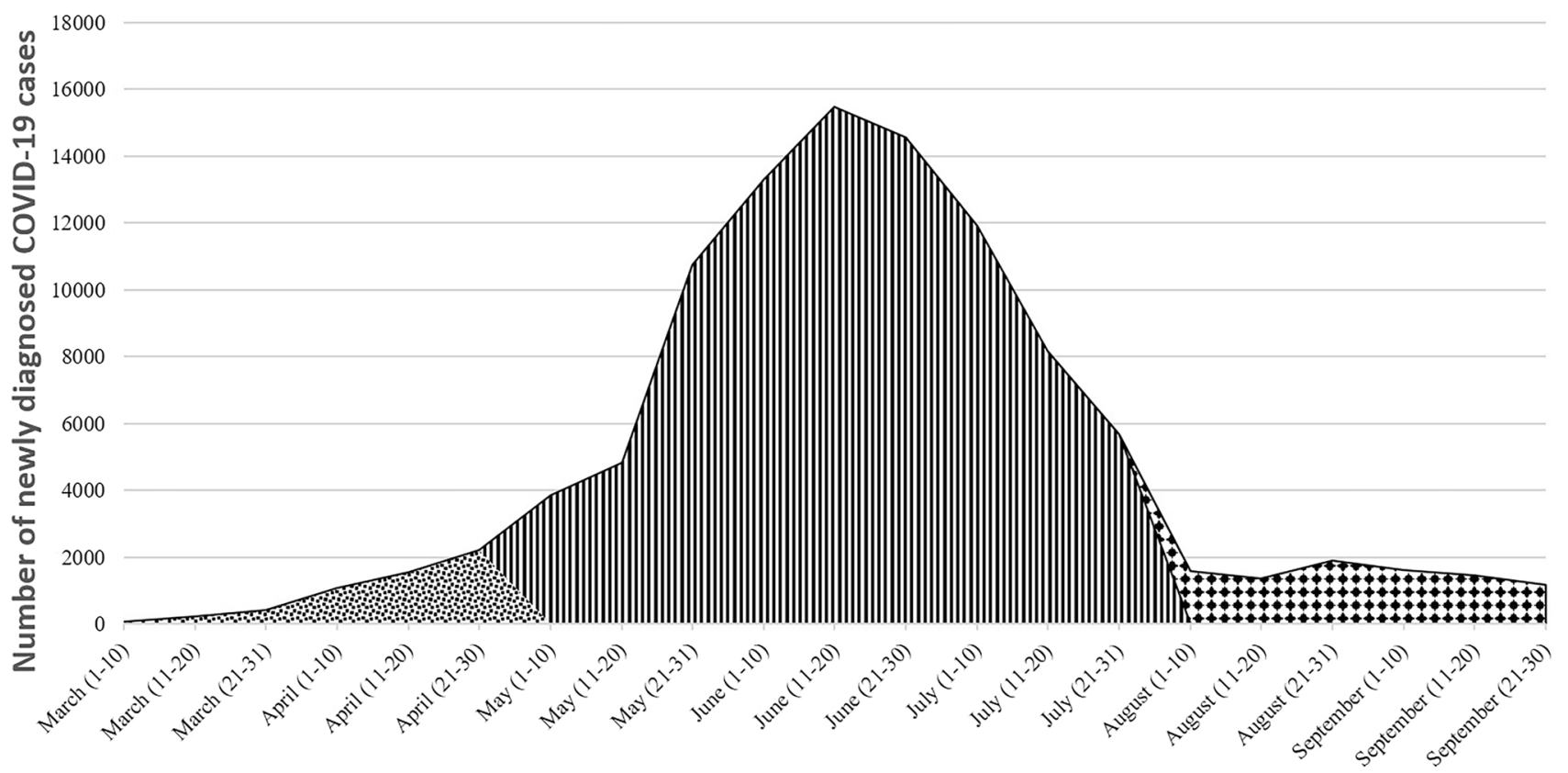

Time intervals of the first wave of COVID-19 pandemic in Egypt

\section{* First Interval III Second Interval $\$$ Third Interval}

Fig. 1 Area chart represents the time intervals of the study period and the distribution of newly confirmed cases infected with COVID-19 


\section{Results}

The study group consisted of 13 patients, 7 males and 6 females. Their age ranged from 3 to 12 years (mean: $5^{7} / 12$ years). The causative pathology was corrosive ingestion in 9 cases, peptic stricture in 2 cases, anastomotic stricture after primary repair of esophageal atresia in one case and congenital stricture in one case. Seven patients presented with long strictures, while six patients had short strictures. Strictures were present in the cervical esophagus in one patient, lower esophagus in two patients, and in the mid esophagus in 10 patients. During the study period, 19 dilatation sessions were performed for these patients (1.46 dilatation sessions per patient) (Table 1).

In the study group, 12 dilatation sessions (63.2\%) were performed in the third time interval. The lowest number of dilatation sessions were performed in the second interval; three sessions in three months. Moreover, nine dilatation sessions were performed in September, while there were no dilatation sessions in June. Eight cases (61.5\%) were recorded with deterioration in the degree of dysphagia score; one case was recorded during the second interval in July, while seven patients were recorded during the third interval. All of them developed dysphagia after alkali ingestion. Three cases were grade 4 , three cases were grade 3 and two cases were grade 2. Difficult dilatation was experienced in three cases; the combined balloon and bougie technique [5] was used in two cases, while a retrograde dilatation [6] approach was applied in one case. Failed dilatation was documented in two cases due to the development of complete obstruction.
Further interventions were required in four patients (30.8\%). Two cases were referred for colon interposition after the failure of the endoscopic dilatation of the esophagus in both cases. A gastrostomy was performed in one case due to severe deterioration in dysphagia score, while a rigid esophagoscope was used in one case to dislodge an impacted food bolus.

The control group included 29 patients. Their age ranged from $2 \%_{12}$ to 13 years (mean: $5^{10} /_{12}$ years). They were subjected to 98 dilatation sessions (3.38 dilatation sessions per patient). Compared to the study group, the highest number of dilatation sessions were in June, with the lowest numbers in September (Fig. 2).

During 2019, esophageal substitution surgery was performed in six patients. None of them qualified as candidates for the endoscopic dilatation protocol of the esophagus. Four cases had esophagostomy and gastrostomy due to long gap esophageal atresia, while two cases had caustic strictures which were not amenable to dilatation due to the presence of impassable strictures.

Eight cases were included in both, the study and the control groups. Three of them presented to the emergency room during the study period with grade 4 dysphagia. Impacted food bolus over a short stricture was the cause of presentation in one patient, while complete obstruction was found in two patients who were known to have long strictures.
Table 1 Case burden and distribution of dilatation sessions during the study period

\begin{tabular}{|c|c|c|c|c|c|c|c|c|}
\hline \multirow[t]{2}{*}{ Patients } & \multicolumn{2}{|c|}{ First interval } & \multicolumn{3}{|c|}{ Second interval } & \multicolumn{2}{|c|}{ Third interval } & \multirow[t]{2}{*}{ Total } \\
\hline & March & April & May & June & July & August & September & \\
\hline 1 & 1 & & & & & & & 1 \\
\hline 2 & & 1 & & & & & 1 & 2 \\
\hline 3 & & 1 & & & & & 2 & 3 \\
\hline 4 & & 1 & & & & & & 1 \\
\hline 5 & & & 1 & & & & 1 & 2 \\
\hline 6 & & & & & 1 & & & 1 \\
\hline 7 & & & & & 1 & 1 & & 2 \\
\hline 8 & & & & & & 1 & 1 & 2 \\
\hline 9 & & & & & & 1 & & 1 \\
\hline 10 & & & & & & & 1 & 1 \\
\hline 11 & & & & & & & 1 & 1 \\
\hline 12 & & & & & & & 1 & 1 \\
\hline 13 & & & & & & & 1 & 1 \\
\hline \multirow[t]{2}{*}{ Total } & 1 & 3 & 1 & 0 & 2 & 3 & 9 & 19 \\
\hline & 4 & & 3 & & & 12 & & \\
\hline
\end{tabular}




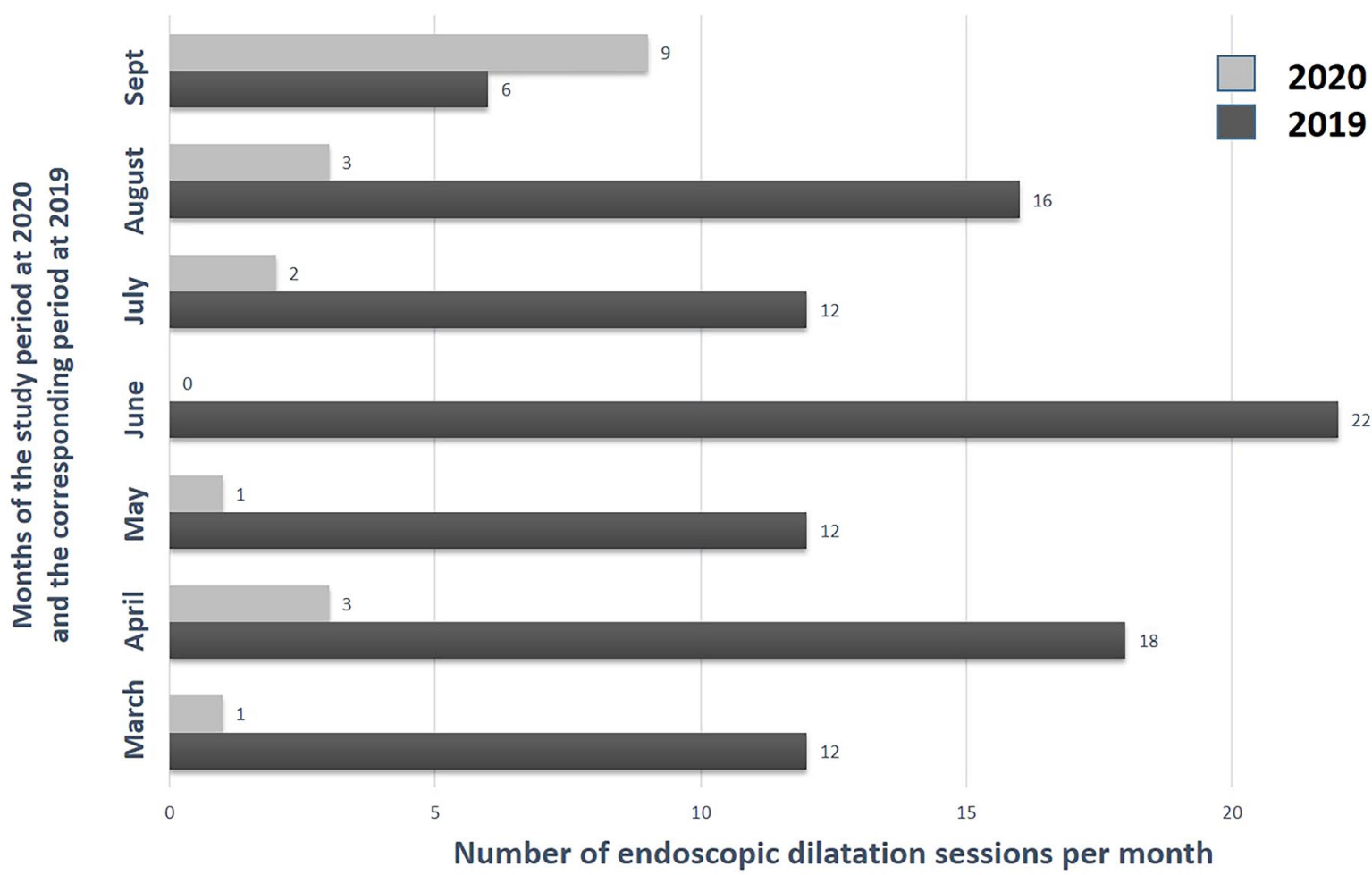

25

Fig. 2 Bar chart represents the difference in the number of endoscopic dilatation sessions per month between the study group and the control group

\section{Discussion}

The initial wave of the COVID-19 pandemic was less prevalent among children than adults [7, 8]. Reports stated that children were affected in a different way compared to adults $[9,10]$. Approximately $90 \%$ of affected children and adolescents were asymptomatic or encountered mild or moderate symptoms [11-13]. According to the Chinese Centre for Disease Control, $15.8 \%$ of children infected with COVID19 were asymptomatic compared to $1 \%$ of infected adults [7, 14]. Also, symptomatic cases in the pediatric population had atypical presentations. With regards to surgery, it has been reported that the invasive nature of surgery may possibly contribute to the transmission of COVID-19 between patients and medical staff [15]. Furthermore, perioperative stress and associated immune dysregulation may exacerbate the clinical condition in COVID-19 patients [16].

Many reports highlighted the impact of COVID-19 on pediatric surgery [17-19]. In our Institute, all outpatient and elective services that could be safely postponed, were temporarily suspended during the lockdown in response to the initial wave of COVID-19. This was done with the intention to limit nosocomial disease transmission and redirect human resources and logistics to handle the increasing number of COVID-19 patients. These were almost the same measures that were applied all around the world in response to the pandemic [17].

In relation to the high percentage of asymptomatic cases and the atypical presentation in the pediatric population, the hospital adopted a policy of screening all children admitted during the epidemic period. An alternative pathway was created for confirmed or suspected COVID-19 cases, in addition to restrictions placed on outside visitors. After hospital discharge, follow-up was limited to phone calls in order to reduce unnecessary exposure as long as the postoperative course was acceptable. Screening remained mandatory for primary caregivers accompanying young children in order to keep a check and reduce exposure to hospital staff.

The results of the proposed study showed a $55.17 \%$ reduction in case burden during the lockdown period compared to the same period in the previous year. The total number of dilatation sessions in the study group were reduced by $80.6 \%$ when compared to the control group. In the same time period, there was a significant decrease in the number of dilatation sessions per patient in the study group compared to the control group (1.46 vs 3.38 , respectively).

All dilatation sessions performed during the first and second intervals of the lockdown, were performed as urgent measures or emergency procedures. It was observed that there was a catch-up in case burden as well as dilatation 
sessions associated with the reduction of procedures during the lockdown.

Parents and caregivers expressed fear that COVID-19 infection may be transmitted to them or their children from the hospital. In April 2020, Professor Russell Viner, President of Royal College of Paediatrics and Child health, expressed concern about children with diseases not related to COVID-19, who were very ill or died because their parents or caregivers had concerns about going to hospitals [20]. In the authors' view, the uncertainty, the lack of standardized protocols, and the general orientation that people should stay home and maintain social distancing resulted in increasing anxiety.

Children with esophageal strictures, who were treated with endoscopic dilatation of the esophagus are among cases that should not be postponed. This is important to maintain their nutritional status and also to preseve their native esophagus. In the current study, two children with caustic esophageal strictures lost their native esophagus due to the development of complete obstruction. It should be taken into account that among the various benign pathologies of esophageal strictures, caustic strictures should not be neglected, and any delay in diagnosis or discontinuation of treatment could lead to unfavourable consequences. Our records showed that four out of eight patients who experienced deterioration in their dysphagia score during the study period were responding well to the endoscopic dilatation during 2019. In the same context, three out of four patients who needed further interventions in the study group started their dilatation program at least one year before the onset of COVID-19. This data suggested that the interruption of their dilatation program was the cause of their deterioration rather than the pathology itself. The esophagus of the two patients who had substitution surgery due to complete obstruction during the COVID-19 era, were initially dilatable during 2019 when they were adhered to the dilatation program.

Although the current study is limited to a single center, along with a specific pathology, its results may reflect the general state of pediatric surgical services during the initial wave of the COVID-19 pandemic. Since the duration of the pandemic is unknown, hospitals should implement strict measures to avoid similar morbidities during the subsequent waves.

European Society for Paediatric Gastroenterology, Hepatology and Nutrition (ESPGHAN) stated that elective upper gastrointestinal endoscopy procedures should be suspended during the COVID-19 pandemic [21]. The present study denoted the importance to embrace a patient-based approach; with precise identification of the group of high-risk patients in each pathology and actively following-up by phone calls carried out by medical staff. Parents and caregivers should be reassured that hospitals are dedicating resources and creating safe pathways to protect uninfected children as well as their companions from COVID-19.

\section{Conclusion}

Interruptions in the routine endoscopic dilatation program for patients with benign esophageal strictures because of COVID-19 pandemic has negatively affected children and resulted in unfavorable outcome in the high-risk group.

Funding No funding was received for conducting this study.

\section{Declarations}

Conflict of interest The authors have no relevant financial or non-financial interests to disclose.

\section{References}

1. WHO Director-General's opening remarks at the media briefing on COVID-19-11 March 2020. WHO Director-General's opening remarks at the media briefing on COVID-19-11 March 2020. Accessed 15 Jan 2021

2. El-Asmar KM, Allam AM (2020) Predictors of successful endoscopic management of caustic esophageal strictures in children: When to stop the dilatations? J Pediatr Surg S00223468(20):30595-30599. https://doi.org/10.1016/j.jpedsurg.2020. 08.017

3. Parthipun A, Diamantopoulos A, Shaw A, Dourado R, Sabharwal T (2014) Self-expanding metal stents in palliative malignant oesophageal dysplasia. Ann Palliat Med 3(2):92-103. https://doi. org/10.3978/j.issn.2224-5820.2014.04.03

4. EgyptCare, https://www.care.gov.eg/EgyptCare/News/Browse. aspx?cid=1. Accessed 23 Jan 2021

5. El-Asmar KM, Youssef AA, Abdel-Latif M (2021) The effectiveness of combined balloon and bougie dilatation technique in children with impassable esophageal stricture. J Laparoendosc Adv Surg Tech A. https://doi.org/10.1089/lap.2020.0926

6. El-Asmar KM, Youssef AA (2019) Retrograde endoscopic dilatation for difficult caustic esophageal strictures: Feasibility and effectiveness. J Pediatr Surg 54(9):1953-1957. https://doi.org/10. 1016/j.jpedsurg.2018.12.016

7. Wu Z, McGoogan JM (2020) Characteristics of and important lessons from the coronavirus disease 2019 (COVID-19) outbreak in china: summary of a report of 72314 cases from the Chinese center for disease control and prevention. JAMA 323(13):12391242. https://doi.org/10.1001/jama.2020.2648

8. Guan WJ, Ni ZY, Hu Y, Liang WH, Ou CQ, He JX, Liu L, Shan H, Lei CL, Hui DSC, Du B, Li LJ, Zeng G, Yuen KY, Chen RC, Tang CL, Wang T, Chen PY, Xiang J, Li SY, Wang JL, Liang ZJ, Peng YX, Wei L, Liu Y, Hu YH, Peng P, Wang JM, Liu JY, Chen Z, Li G, Zheng ZJ, Qiu SQ, Luo J, Ye CJ, Zhu SY, Zhong NS (2020) China medical treatment expert group for COVID-19. Clinical characteristics of coronavirus disease 2019 in China. N Engl J Med. 382(18):1708-1720. https://doi.org/10.1056/NEJMo a2002032 
9. Jiehao C, Jin X, Daojiong L, Zhi Y, Lei X, Zhenghai Q, Yuehua Z, Hua Z, Ran J, Pengcheng L, Xiangshi W, Yanling G, Aimei X, He T, Hailing C, Chuning W, Jingjing L, Jianshe W, Mei Z (2020) A case series of children with 2019 novel coronavirus infection: clinical and epidemiological features. Clin Infect Dis 71(6):1547-1551. https://doi.org/10.1093/cid/ciaa198

10. Xia W, Shao J, Guo Y, Peng X, Li Z, Hu D (2020) Clinical and CT features in pediatric patients with COVID-19 infection: Different points from adults. Pediatr Pulmonol 55(5):1169-1174. https:// doi.org/10.1002/ppul.24718

11. Ludvigsson JF (2020) Systematic review of COVID-19 in children shows milder cases and a better prognosis than adults. Acta Paediatr 109(6):1088-1095. https://doi.org/10.1111/apa.15270

12. Hong H, Wang Y, Chung HT, Chen CJ (2020) Clinical characteristics of novel coronavirus disease 2019 (COVID-19) in newborns, infants and children. Pediatr Neonatol 61(2):131-132. https://doi. org/10.1016/j.pedneo.2020.03.001

13. Qiu H, Wu J, Hong L, Luo Y, Song Q, Chen D (2020) Clinical and epidemiological features of 36 children with coronavirus disease 2019 (COVID-19) in Zhejiang, China: an observational cohort study. Lancet Infect Dis 20(6):689-696. https://doi.org/10.1016/ S1473-3099(20)30198-5

14. Lu X, Zhang L, Du H, Zhang J, Li YY, Qu J, Zhang W, Wang Y, Bao S, Li Y, Wu C, Liu H, Liu D, Shao J, Peng X, Yang Y, Liu Z, Xiang Y, Zhang F, Silva RM, Pinkerton KE, Shen K, Xiao H, Xu S, Wong GWK (2020) Chinese Pediatric Novel Coronavirus Study Team. SARS-CoV-2 infection in children. N Engl J Med 382(17):1663-1665. https://doi.org/10.1056/NEJMc2005073

15. Dedeilia A, Esagian SM, Ziogas IA, Giannis D, Katsaros I, Tsoulfas $G$ (2020) Pediatric surgery during the COVID-19 pandemic.
World J Clin Pediatr 9(2):7-16. https://doi.org/10.5409/wjcp.v9. i2.7

16. Besnier E, Tuech JJ, Schwarz L (2020) We asked the experts: COVID-19 outbreak: is there still a place for scheduled surgery? "Reflection from pathophysiological data." World J Surg 44(6):1695-1698. https://doi.org/10.1007/s00268-020-05501-6

17. Zhou Y, Xu H, Li L, Ren X (2020) Management for patients with pediatric surgical disease during the COVID-19 epidemic. Pediatr Surg Int 36(6):751-752. https://doi.org/10.1007/ s00383-020-04656-6

18. Davenport M, Pakarinen MP, Tam P, Laje P, Holcomb GW 3rd (2020) From the editors: The COVID-19 crisis and its implications for pediatric surgeons. J Pediatr Surg 55(5):785-788. https:// doi.org/10.1016/j.jpedsurg.2020.04.009

19. Polites SF, Azarow KS (2020) Perspectives on pediatric appendicitis and appendectomy during the severe acute respiratory syndrome coronavirus 2 pandemic. J Laparoendosc Adv Surg Tech A 30(4):356-357. https://doi.org/10.1089/lap.2020.0197

20. Royal College of Paediatrics and Child Health. COVID-19 - statement on delayed presentations. https://www.rcpch.ac.uk/newsevents/news/covid-19-statement-delayed-presentations. Accessed 17 Jan 2021

21. European Society for Paediatric Gastroenterology, Hepatology and Nutrition (ESPGHAN) - GI Endoscopy in Children and COVID-19 Pandemic, https://www.espghan.org/knowledgecenter/COVID-19-informational-statements. Accessed 30 Mar 2021 